\title{
Compressible Flow Tables for Engineers
}

with appropriate computer programs, for estimating property changes caused by friction heat transfer and/or shock waves

James Palmer, Kenneth Ramsden and Eric Goodger

Senior Lecturers

The School of Mechanical Engineering

Cranfield Institute of Technology

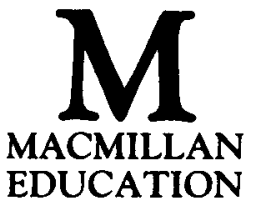


(c) J. R. Palmer, K. W. Ramsden and E. M. Goodger 1987

All rights reserved. No reproduction, copy or transmission of this publication may be made without written permission.

No paragraph of this publication may be reproduced, copied or transmitted save with written permission or in accordance with the provisions of the Copyright Act 1956 (as amended).

Any person who does any unauthorised act in relation to this publication may be liable to criminal prosecution and civil claims for damages.

First published 1987

Published by MACMILLAN EDUCATION LTD

Houndmills, Basingstoke, Hampshire RG21 2XS

and London

Companies and representatives

throughout the world

ISBN 978-0-333-44764-2

DOI 10.1007/978-1-349-09724-1

Macmillan title of related interest

E. M. Goodger Principles of Engineering Thermodynamics (second edition) 


\section{Contents}

Introduction 1

Notation 2

Unit Conversions 3

Typical Values of Specific Heat Ratio and Gas Constant 4

1. Isentropic Flow: Introduction 5

Program 6

$\begin{array}{ll}\text { Tables } & 7\end{array}$

2. Isothermal Flow: Introduction 31

$\begin{array}{ll}\text { Program } & 32\end{array}$

Tables $\quad 33$

3. Rayleigh Flow: Introduction 45

$\begin{array}{ll}\text { Program } & 46\end{array}$

$\begin{array}{ll}\text { Tables } & 47\end{array}$

4. Fanno Flow: Introduction 65

$\begin{array}{ll}\text { Program } & 66\end{array}$

$\begin{array}{ll}\text { Tables } & 67\end{array}$

5. Plane Shock Wave: $\quad$ Introduction 79

$\begin{array}{ll}\text { Program } & 81\end{array}$

$\begin{array}{lr}\text { Tables } & 84\end{array}$

6. Prandtl-Meyer Expansion: Introduction 89

$\begin{array}{ll}\text { Program } & 90\end{array}$

$\begin{array}{ll}\text { Tables } & 91\end{array}$ 